\title{
A QUADRATIC LOWER BOUND FOR COLOURFUL SIMPLICIAL DEPTH
}

\author{
TAMON STEPHEN AND HUGH THOMAS
}

\begin{abstract}
We show that any point in the convex hull of each of $(d+1)$ sets of $(d+1)$ points in $\mathbb{R}^{d}$ is contained in at least $\left\lfloor(d+2)^{2} / 4\right\rfloor$ simplices with one vertex from each set.
\end{abstract}

\section{INTRODUCTION}

Given a set $S$ of points in $\mathbb{R}^{d}$ and an additional point $p$, the simplicial depth of $p$ with respect to $S$, denoted $\operatorname{depth}_{S}(p)$, is the number of closed $d$-simplices generated from points of $S$ that contain $p$. This can be viewed as a statistical measure of how representative $p$ is of $S[6]$. In [5] the authors consider configurations of $d+1$ points in each of $d+1$ colours in $\mathbb{R}^{d}$. They define the colourful simplicial depth of $p$ with respect to a configuration $\mathbf{S}$, denoted $\operatorname{depth}_{\mathbf{S}}(p)$, as the number of $d$-simplices containing $p$ generated by sets of points from $\mathbf{S}$ that contain one point of each colour.

Given a configuration $\mathbf{S}=\left\{S_{1}, \ldots, S_{d+1}\right\}$ the core of the configuration is the intersection of the convex hulls of the individual colours, i.e. $\bigcap_{i=1}^{d+1} \operatorname{conv}\left(S_{i}\right)$. Define:

$$
\mu(d)=\min _{\text {configurations } \mathbf{S} \text { in } \mathbb{R}^{d}, p \in \operatorname{core}(\mathbf{S})} \operatorname{depth}_{S}(p)
$$

The quantity $\mu(d)$ was introduced in [5]. In that paper, it was shown that $2 d \leq \mu(d) \leq d^{2}+1$, and conjectured that $\mu(d)=d^{2}+1$. In this paper we prove

Theorem 1. $\mu(d) \geq\left\lfloor(d+2)^{2} / 4\right\rfloor$.

In particular, this shows that $\mu(d)$ is quadratic. The quantity $\mu(d)$ is used in bounding the depth of a monochrome simplicial median (i.e. point of maximum simplicial depth) for $n$ points in $\mathbb{R}^{d}$ via the method of Bárány [1] as described in [5]. We remark also that in optimization, $\mu(d)$ represents the minimum number of solutions to the colourful linear programming feasibility problem proposed in [3] and discussed in [4].

Both authors were supported by NSERC Discovery grants. Additionally, T. Stephen was supported by DFG FG-468 and the Dynamical Systems research focus at the University of Magdeburg. 


\section{PRELiminaries}

We consider only configurations that have a non-empty core. Since we compute depths using closed simplices, degeneracies that cause $p$ to lie on the boundary of a colourful simplex can only increase the colourful simplicial depth by allowing $p$ to lie in different simplices with disjoint interior. Thus, since we are minimizing, we can assume that the core is full-dimensional and the points of $\mathbf{S}$ lie in general position in $\mathbb{R}^{d}$.

We also assume without loss of generality that the minimum in Equation (1) is attained at the origin, $p=\mathbf{0}$. We note that if some point in $\mathbf{S}$ is $\mathbf{0}$ then we are done since all the $(d+1)^{d}$ colourful simplices using this point contain $\mathbf{0}$. Thus we can rescale the non-zero points of $\mathbf{S}$ so that they lie on the unit sphere, $\mathbb{S}^{d} \subset \mathbb{R}^{d}$. Since the coefficients in a convex combination expressing $\mathbf{0}$ can also be rescaled, this does not affect which colourful simplices contain $\mathbf{0 .}$

Indeed, we observe that the colourful set $\left\{x_{1}, \ldots, x_{d+1}\right\}$ generates a colourful simplex containing $\mathbf{0}$ exactly when the antipode $-x_{d+1}$ of $x_{d+1}$ lies in cone $\left(x_{1}, \ldots, x_{d}\right)$, a pointed cone with vertex $\mathbf{0}$. Our strategy will be to understand how $\mathbb{S}^{d}$ can be covered by $d$-coloured simplicial cones, that is, cones that are generated by $d$ points of different colours. In this vein we can define the $D$-depth of a point of colour $i$ to be the number of $d$-coloured simplicial cones of colours $D=\{1, \ldots, \hat{i}, \ldots, d+1\}$ containing the point. We remark that the $D$-depth of any point is at least one. This follows from the result in [1] that every point in a colourful configuration with $\mathbf{0}$ in its core is among the generators of at least one colourful simplex containing $\mathbf{0}$.

Let $e_{1}, \ldots, e_{d}$ be the standard coordinate unit vectors in $\mathbb{R}^{d}$. Recall that the standard cross-polytope is $\operatorname{conv}\left( \pm e_{1}, \ldots, \pm e_{d}\right)$. We will now define a condition on $2 n$ points that means that they "look like" the vertices of a standard crosspolytope, with $\pm e_{i}$ coloured with colour $i$.

Definition 2. A collection of 2 points in each of $d$ colours is said to be in $d e-$ formed cross position if the $2^{d}$ different $d$-coloured simplicial cones generated by the points cover $\mathbb{R}^{d}$.

Note that some of the $d$-coloured simplicial cones generated by the points in deformed cross position may overlap substantially (not just along boundaries). We conclude with the following Lemma, which is proved in Section 3.1.

Lemma 3. If the colourful simplicial depth of $\mathbf{0}$ is less than $d^{2}+d$, then for any choice of a set $D$ of $d$ colours, there must exist a subset of $\mathbf{S}$ in deformed cross position, the colours of whose vertices are given by $D$. 


\section{Proof of Theorem 1}

Assume that the colourful simplicial depth of $\mathbf{0}$ is less than $d^{2}+d$, so that the lemma applies.

Choose a set of points $P_{1}$ in deformed cross position on the colours $\{2, \ldots, d+$ $1\}$. Pick a point $v$ from $\mathbf{S}$ with colour 1 . Its antipode is in at least one $\{2, \ldots, d+1\}$-coloured simplicial cone generated by vertices of $P_{1}$. The vertices of that cone together with $v$ yield a colourful simplex containing $\mathbf{0}$. This procedure yields $d+1$ colourful simplices, one for each element of $\mathbf{S}$ with colour 1.

Now choose a set of points $P_{2}$ in deformed cross position on the colours $\{1,3, \ldots, d+1\}$. Let $v$ be a point from $\mathbf{S}$ with colour 2 which does not appear in $P_{1}$. There are $d-1$ of these. As before, each of these points, together with some vertices from $P_{2}$, generate a colourful simplex containing $\mathbf{0}$. Since we are using vertices of colour 2 which were not used in the first step, the colourful simplices generated at this step are distinct from those generated at the first step. This yields $d-1$ colourful simplices.

Repeat this procedure, at the $i$-th step choosing points in deformed cross position on the colours $\{1, \ldots, \hat{i}, \ldots, d+1\}$, and then considering those vertices of colour $i$ which have not appeared in any $P_{j}$ for $j<i$. This gives $d+1-2(i-1)$ new colourful simplices. Hence the total number of colourful simplices produced is at least: $(d+1)+(d-1)+\cdots=\left\lfloor(d+2)^{2} / 4\right\rfloor$ as desired.

Remark 4. This improves the lower bound of $2 d$ from [5] starting at $d=4$.

Remark 5. The authors have recently learned that Bárány and Matoušek independently found a quadratic lower bound for $\mu(d)$ [2]. Their bound is $\mu(d) \geq \frac{1}{5} d(d+1)$. They also give a lower bound of $3 d$ if $d>2$ which exceeds $(d+2)^{2} / 4$ when $d=3,4,5,6,7$.

3.1. Proof of Lemma 3. Without loss of generality, let $D=\{1, \ldots, d\}$. Consider the $D$-depth of a point in $\mathbb{S}^{d}$. If every point were of $D$-depth at least $d$, then wherever the points coloured $d+1$ are, each of their antipodes is in at least $d D$-coloured simplicial cones, and thus the depth of $\mathbf{0}$ is at least $d^{2}+d$.

Assuming the colourful simplicial depth of $\mathbf{0}$ is less than $d^{2}+d$, there is some point $x \in \mathbb{S}^{d}$ which is in no more than $d-1 D$-coloured cones. Thus, we can choose a set of points $w_{1}, \ldots, w_{d}$ such that $w_{i}$ is of colour $i$ and generates no $D$-coloured cone containing $x$. Let $z_{1}, \ldots, z_{d}$ be the vertices of some $D$-coloured cone containing $x$, with $z_{i}$ of colour $i$.

We claim that $P=\left\{z_{i}\right\} \cup\left\{w_{i}\right\}$ is in deformed cross position. Let $\mathbb{P}^{d}$ be the union of $d$-coloured simplices on the set $P$. Consider the map $f$ which maps 
$\mathbb{P}^{d}$ to $\mathbb{S}^{d}$ by $x \rightarrow x /\|x\|$. We want to show that this map is onto. Suppose otherwise. Let $X$ be the simplex of $\mathbb{P}^{d}$ whose vertices are $\left\{z_{1}, \ldots, z_{d}\right\}$. Let $Y$ be the union of the other simplices of $\mathbb{P}^{d}$. Let $Z=X \cap Y$ be the boundary of $X$.

Let $A$ be the intersection of $\mathbb{S}^{d}$ with the $D$-coloured cone generated by the $\left\{z_{i}\right\}$. Let $B$ be the boundary of $A$.

By definition, $f(X)=A$. Thus, if $f$ is not onto, there is some point $y \notin A$ such that $y$ is not in the image of $f$. Also observe that $x \notin f(Y)$, by our choice of points $\left\{w_{i}\right\}$.

Now, define a map $\pi$ which retracts $\mathbb{S}^{d} \backslash\{x, y\}$ onto $B$. Clearly, restricted to $Z,\left.(\pi \circ f)\right|_{Z}=\left.f\right|_{Z}$ is a homeomorphism, and generates the non-zero homology of $B$. But $\pi \circ f: Y \rightarrow B$ shows that $\left.(\pi \circ f)\right|_{Z}$ is null-homotopic, which is a contradiction.

Thus $f$ must be onto, and our set of points is in deformed cross position.

\section{Acknowledgments}

The authors would like to thank the referees for comments which improved the presentation of the paper.

\section{REFERENCES}

[1] I. Bárány, A generalization of Carathéodory's theorem, Discrete Math. 40 (1982), no. 2-3, 141-152.

[2] I. Bárány and J. Matoušek, Quadratically many colorful simplices, SIAM Journal on Discrete Mathematics 21 (2007), no. 1, 191-198.

[3] I. Bárány and S. Onn, Colourful linear programming and its relatives, Math. Oper. Res. 22 (1997), no. 3, 550-567.

[4] A. Deza, S. Huang, T. Stephen, and T. Terlaky, The colourful feasibility problem, Submitted, 2005. Revised 2006. Preprint available at: arXivimath.CO/0511749.

[5] _ Colourful simplicial depth, Discrete Comput. Geom. 35 (2006), no. 4, 597-604. arXiv math.CO/0506003

[6] K. Fukuda and V. Rosta, Data depth and maximal feasible subsystems, Graph Theory and Combinatorial Optimization (D. Avis, A. Hertz, and O. Marcotte, eds.), SpringerVerlag, New York, 2005, 37-67.

Department of Mathematics, Simon Fraser University, 8888 University Drive, Burnaby, British Columbia, Canada V5A 1S6.

E-mail address: tamon@sfu.ca

Department of Mathematics and Statistics, University of New Brunswick, Fredericton, New Brunswick E3B 5A3 Canada.

E-mail address: hugh@math.unb.ca 